\title{
Extendable high-speed stimulus generation and recording on the Macintosh computer
}

\author{
SCOTT B. STEINMAN \\ Southern College of Optometry, Memphis, Tennessee.
}

\begin{abstract}
A new visual electrophysiology and eye-movement laboratory was developed with three goals in mind: (1) rapid execution speed for quick acquisition of data from infant subjects, (2) ease of operation by experimenters, and (3) modularity and flexibility. A powerful system composed of two linked Macintosh computers was constructed in which one computer was used solely to generate visual stimuli and the second solely to record subjects' responses, allowing recording to execute at maximum speed without being slowed by stimulus graphics generation. A single program on the recording computer allowed the experimenter to choose the stimulus and recording parameters. The two computers intercommunicated via a local EtherNet network to coordinate recording and signal averaging. This paper presents design principles and specific programming techniques relevant to any Macintosh system that must rapidly acquire physiological recordings while simultaneously displaying sensory stimuli.
\end{abstract}

During the past decade, turnkey electrophysiology recording units have gradually been replaced by personalcomputer-based recording systems (e.g., see Baro \& Lehmkuhle, 1988; de Waal, Reits, Spekreijse, \& Grimbergen, 1983; Torok, 1990; van Norren \& van de Kraats, 1983). Personal-computer-based systems offer flexibility not possible in turnkey-dedicated averaging unitsthe stimulus configurations and analysis techniques are limited only by the imagination of the programmer. The advent of fast personal computers has enabled the use of graphical laboratory data acquisition programming languages for rapid program development. Although these languages provide a useful physiology laboratory tool, their clinical and experimental usefulness has been limited in vision science because they cannot present rapidly changing visual stimuli and acquire and display physiological responses at high sampling rates simultaneously.

This paper describes an extremely flexible infant vision laboratory developed by the author for the Washington University School of Medicine using two MC68040-based Apple Macintosh computers. All of the major hardware components and software tools are available from commercial sources or as freeware on the World-Wide Web. Its modular software system was designed to allow standardized stimuli to be presented for tests of visual function, yet permit easy future expansion to include newly developed tests. The system is presently used to record several classes of electrophysiological measurements

The author wishes to thank Ray Hunter of the St. Louis Children's Hospital for his support of the Infant Vision Laboratory, and Barbara Steinman for her many helpful comments on earlier drafts of this manuscript. Correspondence should be addressed to S. B. Steinman, Department of Biomedical Sciences, Southern College of Optometry, 1245 Madison Ave., Memphis, TN 38104-2222 (e-mail: steinman@ sco.edu). (flash electroretinograms [ERG], pattern ERG, flash visual evoked potentials [VEP], pattern VEP, motion VEP [see Norcia, 1993], binocular "beat" VEP [see Baitch \& Levi, 1988]), oculomotor recordings (optokinetic nystagmus [OKN], pursuit, saccade, fixation stability), and psychophysical measurements (forced-choice preferential looking).

The goal of this paper is not to provide all of the steps required to build an infant vision testing system. Rather, this paper will discuss specific programming issues that had to be solved in order to achieve the design goals of a wide variety of laboratory sensory electrophysiological recording systems. In particular, programming techniques for intercomputer coordination to allow simultaneous realtime visual stimulus display and electrophysiological response recording will be examined in detail. However, it is beyond the scope of this paper to be a tutorial on the particular programming languages used; a background in these programming languages is assumed.

\section{Design Goals and Decisions}

While rapid data acquisition and flexibility are naturally two important goals of any physiological recording system, the present system had additional constrictions that made these goals even more critical. First, the time required to conduct the full test sequence needed to be extremely short since our subjects were very young infants whose attention span is limited in duration. The experimenter therefore had to collect data extremely rapidly for any given test, then quickly proceed to the next test so that as much data as possible could be acquired from the infant. Second, the data acquisition and analysis system had to be run by a single experimenter who also had the responsibility of observing the infants' state of alertness, as well as direct the infants' attention to the stimulus display. This not only reinforced the need for speed of testing but also ease of operation. Finally, the software sys- 
tem needed to be modular and easily extended when new tests of infant vision were introduced.

The first major design decision was to choose the LabVIEW programming language as the language in which to implement the data recording and analysis software. LabVIEW is a visual or iconic programming paradigm, which includes a huge library of data acquisition and data analysis code modules (called virtual instruments, or VIs) that may be incorporated to build electrophysiology or oculomotor recording systems. In addition, LabVIEW's integrated user interface design tools allow the construction of software with a uniform intuitive "front panel" of controls that can be easily operated by a experimenter. The visual programming paradigm allows for rapid application development (RAD) or prototyping in a fraction of the time required to write an equivalent $\mathrm{C} / \mathrm{C}++$ program (e.g., see Steinman \& Carver, 1995).

Unfortunately, while LabVIEW is well suited for developing software for data acquisition and analysis, it is not capable of generating rapid animated stimulus display graphics. In fact, LabVIEW can operate at a very high speed only if it displays recorded data after the data acquisition sequence is completed. Simultaneously controlling visual stimulus display while recording and graphing data is not feasible within the LabVIEW environment. One possible solution that was considered was to call Macintosh ToolBox and QuickDraw routines via external $\mathrm{C}$ language external code modules. Unfortunately, these graphics routines cannot be called in parallel with the data acquisition VIs if rapid execution of the data acquisition code is to be achieved. An additional problem is that stimulus programs for vision research typically involve interrupt-driven code to ensure accurate visual stimulus motion velocity or accurate exposure duration timing.

The simultaneous generation of stimulus graphics and data acquisition is risky on a single machine since the processor interrupts of the data acquisition software and those of the stimulus generation code might interfere with each other, and data samples might be "skipped" during graphics operations or the graphics may "shear" when data samples are acquired. These same interrupt issues may arise even if the data acquisition and stimulus generation are handled by separate programs on a single computer. Furthermore, even if the possibility of conflicting or "missed" interrupts were not an issue, Quickdraw graphics operations on the Macintosh computer are not yet rapid enough to allow simultaneous high-speed electrophysiological data acquisition, data display, and animated stimulus displays on a single computer system.

Therefore, the system design selected was to implement the system on two Macintosh computers: one solely for recording, and the other solely for stimulus display. This would allow each computer to perform its single specialized task at maximum speed - that is, the stimulus display generation would not slow down the rate of data acquisition and data display. This decision to use two computers, in turn, created additional design issues.
In the interest of rapid completion of test sequences for our infant population and the need to constantly monitor the infants, it was imperative that the experimenter interact with only one of the two computers. An experimenter could not realistically set recordings on one computer, launch a stimulus program and set its stimulus parameters on the second computer, then coordinate the data acquisition and stimulus display on both computers all while observing the infant subjects.

More importantly, the two computers could not simply run in isolation. Both the recording program on one computer and the stimulus program on the other computer had to "know" what the other was doing at any given moment. Their operations needed to be tightly synchronized if the two computers were to act as a single unit. This was important not only for coordinating the stimulus presentation at the onset of the data recording but it was even more critical for the time-locked stimuli used for evoked potential recording. How would the two computers intercommunicate?

A design decision was made to control the overall operation of the laboratory software using one Macintosh computer containing an analog data acquisition board. In addition to data acquisition, this computer would also be responsible for data analysis and report generation. The second computer would act as a "slave" to the recording computer - that is, it would act only in response to commands sent to it by the "master" recording computer. This slave computer would be responsible solely for stimulus generation. These two computers will be referred to henceforth as the recording computer and the stimulus computer, respectively. Clearly, two forms of communication were required that would be executed independently. The first was to transmit commands from the recording computer to the stimulus computer. If the recording computer was to be used as a central command station to oversee the operation of the entire computer system, it would need to "tell" the stimulus computer what stimulus to display and when. The second form of intercommunication was to maintain tight control over the timing of the sequence of events during data acquisitionthat is, (1) trigger or sync signals to initiate analog signal acquisition sampling, to initiate signal averaging sweeps, or to indicate the completion of the data acquisition period, and (2) a "ready" signal to indicate that the stimulus program had completed all of the preprocessing required to display a visual stimulus. Digital I/O lines were used to transmit these signals.

Finally, two more decisions related to programming language had to be made. The first was which programming language to use for the stimulus generation software. As stated above, the LabVIEW language is not optimal for the programming of rapid or interrupt-driven animated graphics. A second constraint was that this language had to support programming of intercomputer communication through AppleEvents, the Macintosh's standard interprogram communication protocol. LabVIEW has some capabilities for sending AppleEvents 
(with provisos to be mentioned below), but it has extremely limited abilities to respond to AppleEvents. Because of its abilities to overcome these constraints, the $\mathrm{C}++$ programming language was chosen for developing the stimulus display programs. $\mathrm{C}++$ not only allows for the rapid interrupt code required for accurately timed stimulus display palette or frame animation (see Baro \& Hughes, 1991, Steinman, 1993, and Steinman \& Nawrot, 1992, for such graphics techniques) but is also well suited for handling AppleEvents. In addition, the use of $\mathrm{C}++$ allowed the reuse of graphics, digital $\mathrm{l} / \mathrm{O}$, timing interrupt, and experiment control code libraries written previously by the author, which could in turn be reused for future programs.

Although AppleEvents would be involved in the interprogram communication between the data acquisition and stimulus display computers, they could not be used uniformly throughout the software due to limitations in LabVIEW's interprogram communication code, to be discussed below. One possible solution to this problem would be to use AppleScript to transmit scripts containing sequences of AppleEvent commands. A decision was made to forego AppleScript, even though the Macintosh operating system (System 7.0 or higher) provides builtin AppleScript support. For reasons to be discussed below, the interprogram communication was implemented with the Frontier scripting language (Userland Software Incorporated, Redwood City, CA; now available as freeware on the World-Wide Web at http://www.scripting. com/frontier/).

\section{Hardware Components}

The computer hardware system was composed of two Macintosh Quadra computers (Apple Computer, Cupertino, CA). The recording computer was configured with $20 \mathrm{MB}$ of RAM, a 128-MB magneto-optical drive for data storage, and a laser printer for hard copies of electrodiagnostic and oculomotor data. Data acquisition was performed with a high-speed analog-to-digital (A/D) converter board (NB-MIO-16XH-42, National Instruments, Austin, TX).

The stimulus computer was equipped with $8 \mathrm{MB}$ of RAM and a video scan converter (CVLink, DisplayTech Incorporated, Concord, CA) for display of visual stimuli on a 60-in. diagonal television monitor (VS-60VF2, Mitsubishi Electronics America Incorporated, Cypress, CA). A digital input-output (DIO) interface provided control signals to a digital to analog (D/A) converter that presented sinusoidal flicker for binocular beat VEPs via sets of light-emitting diodes embedded in a pair of goggles.

The two computers were connected in two ways. The first connection was composed of DIO interfaces in each computer (NB-DIO-96, National Instruments) joined by a ribbon cable, which provided the path for rapid control signals to synchronize the actions of the two computers during the real time. These included a digital trigger line for ERG and VEP recordings and a syne signal that con- trolled stimulus onset and offset, or acted as a "program ready" signal.

The second connection was an EtherTalk network cable between the two computers, but which was not connected to any local area network. This provided the means of intercomputer communication for stimulus control by the recording computer. Why was the more expensive EtherTalk used rather than standard built-in AppleTalk? In initial testing of the software system, it was found that intercomputer communication over an AppleTalk network was too slow. When AppleEvent commands were sent to the stimulus computer, the recording computer often had to wait for a "reply" that indicated that the command was received correctly. An AppleEvent could "time out" while waiting for this reply if the transmission time for the AppleEvent and its reply was too long. This would lock up the data acquisition sequence as the recording computer kept waiting for the reply. The much quicker EtherTalk was the solution to this problem, allowing replies to be received fairly instantaneously.

It could be argued that the EtherNet connection that carried AppleEvents from one computer to the other could be replaced by a simple high baud rate serial line. However, there is one disadvantage to doing so. In the current system, both the LabVIEW recording program and the $\mathrm{C}++$ stimulus display program are not aware of whether they reside on two computers or on a single computer. The use of Frontier as an intermediary for passing commands hides these details. This means that if the present stimulus generation and recording system could be implemented on a single computer in the future, all that would need to be done is to edit one subset of Frontier scripts. Neither the recording software nor the stimulus display software would need rewriting. If a serial line had been used instead, all of the interprogram communication code would need to be completely rewritten if these programs resided on one computer.

\section{Software System Operation}

For a better understanding of the workings of the vision assessment software, the general test sequence for recording from subjects is as follows: The main program is entered, which establishes the connection between the recording and stimulus computers across the EtherTalk network. The two DIO lines are then initialized. At this point, the experimenter selects a test to be run.

A command is transmitted to the stimulus computer to launch a given stimulus display program, a graphics program that will display such targets as a drifting sinewave grating. The stimulus parameters chosen by the experimenter are transmitted to the display program by a second command. The experimenter then clicks on a button on the recording computer screen to start recording, and a digital signal is sent to the stimulus computer to enable the stimulus animation. The display computer returns a digital signal to trigger data acquisition, timelocked to the stimulus display. After data collection is 
completed - that is, a specific number of electrophysiological recording data samples are collected by the recording computer-commands are sent to the stimulus computer to stop the stimulus presentation, then quit the stimulus display program. Data analysis is performed off line at a later time so that additional tests with different stimulus parameters may be run in rapid succession while the subject's (usually an infant) attention may be maintained. The analysis programs are included as modules in the software system and operated in much the same way as the recording modules.

\section{Software Components}

The software system has been designed to be both flexible and modular. It was composed of three major components. The first was implemented in the LabVIEW 3.0 programming language (National Instruments, Austin, TX) on the recording computer. This code was responsible for establishing the AppleEvent communication link between the two computers across the EtherTalk network and initializing the analog and DIO boards. The experimenter selects with a mouse pointing device which test is to be run, and the program enters one of severa: testspecific subprograms for acquiring physiological data. Each subprogram allows the specification of stimulus and recording parameters, as well as starting, pausing, or aborting data acquisition. A uniform user interface across all tests simplifies operation of the tests and permits the examiner to devote more attention to observing the subject's alertness and behavior.

Visual stimuli are presented via the second component of the software, a collection of very small programs that were written in the $\mathrm{C}++$ programming language (CodeWarrior $\mathrm{C}++$, Metrowerks Incorporated, Mooers, NY; Resorcerer, Mathemaesthetics Incorporated, Chestnut Hill, MA). These programs take advantage of a reusable code library of drawing and animation routines developed by the author (SMS Technologies, Ann Arbor, MI) that allows both palette animation for drifting and counterphase gratings (Baro \& Hughes, 1991) and frame animation for moving complex shapes as pursuit stimuli (Steinman, 1993; Steinman \& Nawrot, 1992). These programs perform only three chores: (1) receive AppleEvent commands (to be discussed below), (2) display animated graphics, and (3) read and write to the DIO lines for synchronization with the recording software.

Coordination between stimulus generation and data acquisition was established by the third component of our software system, which sends and receives AppleEvent commands between Macintosh programs (see Apple Computer, 1991), in this case between the data acquisition program on the master (recording) computer and various stimulus programs on the slave (stimulus) computer. The transmission of commands from one computer to the other is mediated by Apple Open Scripting Architecturecompatible scripts in the the Frontier scripting language. While AppleScript, another means of sending such commands, is a built-in component of the Macintosh operating system, there are several performance issues that compelled us to use Frontier instead, as will be discussed below.

\section{Interprogram Communication Problems and Solutions}

Although data acquisition and analysis with LabVIEW are topics worthy of discussion, information on these areas can easily be obtained elsewhere (Johnson, 1994). What we will discuss in the remainder of this paper will be a topic on which little information has been available: the techniques required for rapid simultaneous stimulus graphics and data acquisition. With a novel combination of different programming tools, several problems that are specific to real-time simultaneous visual stimulation and data acquisition have been solved. Specifically, we will present an easy way to synchronize two Macintosh computers to work as a single electrophysiology laboratory device, via software commands and hardware signals passed between.

The first problem that we had to confront and solve dealt with shortcomings in LabVIEW's capacities for interprogram communication. While AppleEvent support is included in LabVIEW, it is mostly specialized as VIs that are used to execute other VIs, such as AESend Run VI, AESend Open, Run, Close VI, AESend Close VI, AESend Abort VI, and AESend VI Active?, or responses to these commands. For programs composed of code other than its own VIs, LabVIEW is more capable of responding to commands than sending them.

A few other AppleEvent-related VIs exist, which are geared toward starting or quitting other programs, but these are not sufficient for laboratory program control. Fortunately, a LabVIEW VI that is often overlooked by programmers just happens to be the one VI that is critical for controlling the operation of the stimulus graphics programs by the recording computer. This VI is called AESend Do Script. As its name implies, it is specialized toward sending scripts--sequences of commands encoded as textual strings. Scripts may be written in any of several languages created for interprogram control on the Macintosh, such as AppleScript (where the commands are sequences of AppleEvents to be executed) or HyperCard. When such scripts are received by a target program, their text is decoded into the series of instructions to be carried out by that program.

The next decision was therefore to choose a specific scripting language. The most commonly used systemwide scripting language on the Macintosh is AppleScript. However, the use of AppleScript presents several obstacles. The first is that while AppleScript is very beneficial for sending sequences of instructions to programs, it is relatively slow, even on rapid PowerPC-based Macintoshes, on which it is executed in emulated 68000 code. AppleScript is therefore not very well suited for programs that need to execute subject test sequences quickly.

A second problem is that AppleScript (as well as AppleEvents themselves) was not primarily designed for sending commands quickly over a network to a second 
computer. To send an AppleScript or AppleEvent across a network, the PPC Browser must be invoked. The PPC Browser is intended to allow users to choose which computer and application should be sent a command, but it also has one shortcoming for our purposes. It provides a level of security on the network. After the computer and program with which to "connect" have been chosen, the operator must enter their user name and password for access to the second computer's program via the User Identity dialog box. While this is useful for preventing unwanted connections across the Internet, it is a severe impediment to our design goals. Every time we need to send an AppleScript or an individual AppleEvent to a different stimulus display program, we will be faced with the PPC Browser and User Identity dialog boxes! This is not only disruptive, but it will slow down our data collection.

A third problem is that if only AppleScripts are transmitted to the stimulus computer in order to initiate a stimulus display, these stimulus programs must be capable of receiving and parsing AppleScripts. This presents a very difficult task for several reasons: (1) AppleScript programming is not entirely intuitive and few example programs exist for learning to do so. It is a difficult enough task that only a relatively small proportion of commercial Macintosh programs are scriptable. (2) AppleScript programming also requires programming knowledge about "aete" resources, special Macintosh resources that identify the AppleEvents that may be "understood" by the receiving program. The steps required for the creation of these resources are not very well documented. (3) In the present software system, this would require adding AppleScript support to each of a dozen small stimulus display programs. While adding AppleScript is a fruitful option for single large commercial programs, the time and effort required to add AppleScript support to several small specialized laboratory programs are simply not cost effective. We have selected an alternative that is simpler, yet overcomes many of the limitations of AppleScript.

That alternative is Frontier, the first system-wide scripting environment released for the Macintosh computer (even before AppleScript), now available as freeware on the World-Wide Web. Although Frontier is not an integrated part of the Macintosh operating system (as is AppleScript), it is widely used by commercial software developers as the scripting language of choice. It can transmit sequences of AppleEvents across a network at least 10 times more rapidly than AppleScript scripts, and it does not require the creation of aete resources.

In our laboratory electrophysiology system, Frontier is installed on both the recording computer and the stimulus computer. When a command must be sent from the LabVIEW program on the recording computer to a visual display program on the stimulus computer, it is first sent as a script from LabVIEW to a "master" copy of Frontier on the recording computer. This Frontier application is "told" to run a script stored within itself that essentially transmits the command contained in the original LabVIEW script across the EtherNet network to a "slave" copy of Frontier on the second computer. This second copy of Frontier translates the command into AppleEvent format and relays the command to the stimulus program, where the command is carried out.

Why use such a circuitous route? Why not just send scripts directly from the LabVIEW recording program to the stimulus display program on the second computer? Two reasons have already been mentioned: (1) Frontier speeds up the transmission of commands across the network, and (2) to avoid the need to add AppleScript-parsing code to each stimulus program. Let us add two more important reasons to use Frontier as an intermediary in passing along commands: (3) Frontier simplifies our programming task by translating the original command from LabVIEW's Do Script VI, which is in textual script format, into a form that can be handled with simple code in the stimulus programs-AppleEvent handlers. (4) Because Frontier itself sends the commands across the EtherNet network, the intrusive PPC Browser and User Identity dialog boxes are avoided during the time-critical portions of data collection.

When the LabVIEW program on the recording computer must find a program on the stimulus computer to which to "connect" and send commands, it connects to the master copy of Frontier on its own machine. Similarly, the slave copy of Frontier on the stimulus computer connects to stimulus programs that reside on its own computer. These two communication paths do not require the PPC Browser or User Identity dialog boxes, since each connection between programs is made within a single machine. The connection between the two machines is made by Frontier itself before the LabVIEW program is executed, by means of its NetFrontier suitea collection of scripts that deals solely with interconnecting two or more copies of Frontier on different computers connected across a network.

Before launching the LabVIEW recording program in an experimental session, a small Frontier script is run on the recording Macintosh that results in the master copy of Frontier on that Macintosh establishing a connection across the network to the slave copy of Frontier on the stimulus Macintosh. This is the only time at which the PPC Browser and User Identity dialog boxes appear. During all subsequent experimental testing, these dialog boxes never reappear, even when the experimenter switches between test types and stimulus types, because the connection across the network has already been established from one copy of Frontier to the other.

It should be noted that we have had to modify the arguments passed to some of the NetFrontier commands to include a second parameter that allows the option of waiting or not waiting for a reply during transmission of a script to the stimulus computer. This is because, by default Frontier waits for a reply from the receiving application. When we send commands to show visual stimuli just as we are about to begin data acquisition, we cannot afford the luxury of waiting for a reply, because this would delay the onset of the data acquisition. It is therefore im- 
perative to have the option of avoiding a reply. We do this in our Frontier scripts by replacing Frontier's appleEvent command, which performs the actual transmission of information across the network, with the similar finderEvent command; finderEvent, by default, does not wait for a response. This command was originally intended for sending scripts from Frontier to the Macintosh System 7's Scriptable Finder, but it also suits our purposes well.

Once the intercomputer connection is made, we launch the LabVIEW recording program and set stimulus and recording parameters prior to data collection. Setting stimulus parameters requires sending commands and data across the EtherTalk network, as dictated by scripts sent from LabVIEW to Frontier within the recording computer. But LabVIEW's script transmission VIs expect to be sending commands by connecting to another program across a network, and we do not want that to occur. We want LabVIEW to communicate only with the copy of Frontier hosted on the same computer, and let this copy of Frontier handle the communication across the network to the stimulus computer. In other words, we have to force LabVIEW to establish a connection directly to the Frontier application within the recording computer.

We take advantage of a LabVIEW PPC Toolkit VI named Get Target ID, which receives the name of the application to find and return its target ID. The target ID is a LabVIEW structure (or "cluster" in LabVIEW terminology) that stores the location of a program that exists on the network. We restrict the search to the copy of Frontier on the same machine hosting the LabVIEW recording program. The target ID returned by this search is used in all subsequent command transmissions to Frontier.

Now that we have examined the framework by which LabVIEW and Frontier communicate between themselves to establish control across a network, we will discuss the special programming steps required to send both commands and data from the LabVIEW recording program to their real target, the $\mathrm{C}++$ stimulus display programs. In the example code to follow, the runStimulus command transmitted from the LabVIEW recording pro- gram to a stimulus program will be explained in detailin particular, the version of this script that is used to set up and execute a counterphase-reversing grating presentation program for pattern VEP recordings. This code is used once the recording and stimulus parameters have been chosen in the LabVIEW program and we are ready to initiate data acquisition in response to the grating stimulus. The LabVIEW program initializes data acquisition, then sends a runStimulus script containing counterphase grating stimulus settings to the Frontier application on the stimulus computer. Frontier then repackages the text contained within this AppleEvent into a format that can be sent to and understood by the copy of Frontier on the stimulus computer-a Frontier script. On receipt of the restructured script, the stimulus Macintosh's Frontier application executes the script, extracts its parameters, and sends the parameters within an AppleEvent to the stimulus display program. An AppleEvent handler in that program retrieves the stimulus settings from the AppleEvent parameters and displays the stimulus after sending a sync signal via the DIO lines to let the recording Macintosh know that it is time to start acquiring data. The net result is that the stimulus program only needs to receive an AppleEvent and its parameters in an easy-toextract format, rather than a textual AppleScript that requires complex code to decipher. In other words, the display program need not "understand" the scripting code output by LabVIEW or the scripts transmitted by Frontier. Such code is difficult to decipher by $\mathrm{C}++$ programs. Rather, it need only understands very basic, low-level AppleEvents, which require a minimum of programming to decode. Our Frontier programming has allowed us to simplify our programming task for each stimulus display program to the point that each program need only be a small skeleton program written in $\mathrm{C}++$, consisting solely of AppleEvent-handling and stimulus generation graphics code. The message passing pathway between the recording and stimulus computers is summarized in Figure 1.

One aspect of this interprogram control that has not yet been discussed is the launching and quitting of the stim-

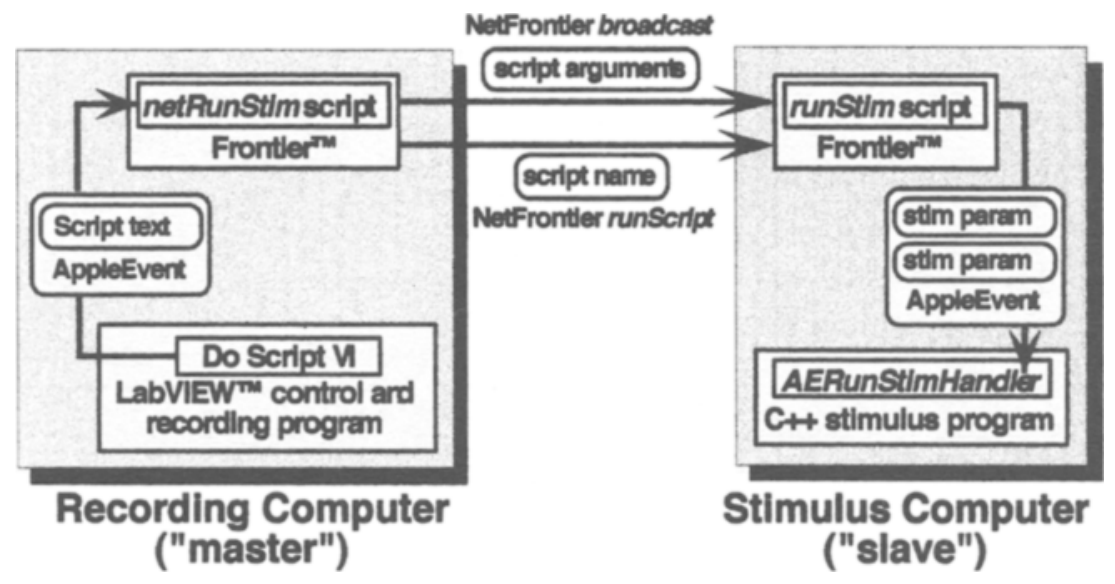

Figure 1. Summary of command transmission from LabVIEW to the $\mathrm{C}++$ stimulus display programs. Frontier translates the script sent by LabVIEW into simpler AppleEvents and transmits them across the EtherTalk network. 
ulus display program prior to and following data acquisition. Frontier simplifies these tasks as well. NetFrontier can instruct the copy of Frontier on the stimulus computer to execute two other Frontier commands. The first is Frontier's launch command, which sends an AppleEvent via the Finder to launch a program. This command finds an application whose signature we supply. The second command, to be used after data collection, is Frontier's quit command, which instructs an executing program to quit itself.

Frontier can therefore be used to handle all aspects of the interprogram communication that is at the core of the laboratory electrophysiology software. Frontier launches the stimulus generation programs, signals each to display stimuli with specific stimulus parameters, then forces them to quit when we no longer need them. The sole purpose of the DIO lines is to ensure the tight time-locking of stimulus display and data recording.

\section{Conclusions}

In the construction of software systems, complex decisions regarding individual aspects of the system design must be made that have serious implications for the design of the remainder of the system. In the present case, the overwhelming need for program execution speed dictated the choice of a dual-computer system and the method of communication between the computers. Such a system could not have been constructed easily without combining the strengths of the LabVIEW iconic laboratory programming language, the Frontier scripting language, and the $\mathrm{C}++$ programming language. The use of LabVIEW shortened the program development cycle considerably. In addition, intercomputer communication of the speed and complexity used here would not have been achieved easily using $\mathrm{C}++$ without the addition of Frontier.

Despite the complexity of this system, the software is easy to operate by the experimenter due to the intuitive user interface imposed by LabVIEW's instrument panel paradigm and the operation of all tests from a single program on the recording computer. More importantly, the system design does not sacrifice modularity and expandability. Adding a new test requires adding only a small skeleton $\mathrm{C}++$ stimulus display program, a few Frontier scripts, and a few LabVIEW VIs to select stimulus parameters, send scripts, and record data. These code modules are very similar in each of the recording, stimulus, and intercommunication programs, largely due to a high degree of code reuse in LabVIEW, Frontier, and C++ libraries for handling AppleEvents and generating animated graphics. Much of the task of creating a new set of tests simply involves duplicating then modifying existing code.

In the future, it may be possible to remove some of the complexity of the system once it is possible to perform all of the stimulus display and recording tasks on a single dual-monitor computer system. The interprogram communication could be restricted to one machine, eliminating the need for the NetFrontier suite: Scripts could be sent from LabVIEW to Frontier and directly translated into
AppleEvents to be received by the stimulus program. Such a system would also be lower in cost and easier to maintain. It is likely that extremely rapid PowerPC processors may eliminate the need for dual dedicated computers. A single extremely rapid PowerPC processor would not only speed up graphics and data acquisition code but would also decrease the time required to process interrupts, reducing the likelihood that graphics and data acquisition interrupts would have mutually deleterious effects. Another important benefit of Frontier will become evident in the future when single PowerPC-based computer systems can be used for simultaneous stimulation and recording. Frontier can use the fast Component Manager of Power Macintoshes for AppleEvent transmission within a single machine, which increases the speed of interprogram communication even more. However, even with the present single-processor technology, we have demonstrated that the creation of powerful, flexible, real-time electrophysiological laboratory software is facilitated when it makes use of innovations found on the Macintosh computer. The same programming principles outlined in this paper may be applied to a wide range of psychology laboratories.

\section{Availability of Code}

The sample code described in this manuscript may be obtained from the author by e-mail at steinman@sco.edu or by anonymous ftp from the MacPsych site.

\section{REFERENCES}

APPle COMPUTER (1991). Inside Macintosh: Interprogram communication. Menlo Park, CA: Addison-Wesley.

BAITCH, L. W., \& LEVI, D. M. (1988). Evidence for nonlinear binocular interactions in human visual cortex. Vision Research, 28, $1139-1143$.

BARO, J. A., \& Hughes, H. C. (1991). The display and animation of fullcolor images in real time on the Macintosh computer. Behavior Research Methods, Instruments, \& Computers, 23, 537-545.

Baro, J. A., \& LeHMKuhle, S. (1988). A software system for recording and analyzing transient evoked potential data with an Apple IIe computer. Behavior Research Methods, Instruments, \& Computers, 20, 515-516.

de WaAl, D. J., Reits, D., Spekreijse, H., \& Grimbergen, C. A. (1983). Implementation of a portable pattern stimulator and VEP/ ERG recording system based on an Apple microcomputer. Documenta Ophthalmologica Proceedings Series, 37, 209-216.

JoHnson, G. W. (1994). LabVIEW graphical programming: Practical applications in instrumentation and control. New York: McGraw-Hill.

NorCIA, A. M. (1993). Improving infant evoked response measurement. In K. Simons (Ed.), Early visual development: Normal and abnormal (pp. 536-552). Oxford: Oxford University Press.

Steinman, S. B. (1993). Simple real-time color frame animation. MacTech, 9(9), 21-35

Steinman, S. B., \& CARver, K. (1995). Visual programming with Prograph CPX. Greenwich, CT: Prentice-Hall, Manning Publications.

Steinman, S. B., \& Nawrot, M. (1992). Real-time color-frame animation for visual psychophysics on the Macintosh computer. Behavior Research Methods, Instruments, \& Computers, 24, 439-452.

ToroK, B. (1990). Microcomputer-based recording system for clinical electrophysiology. Documenta Ophthalmologica, 75, 189-197.

VAN NORREN, D., \& VAN DE KRAATS, J. (1983). Interactive computer program for clinical electrophysiology. Documenta Ophthalmologica Proceedings Series, 37, 193-197.

(Manuscript received October 7, 1996; revision accepted for publication January $31,1997$. 